\title{
Performance evaluation of high-detectivity p-i-n infrared photodetector based on compressively-strained Geo.964Sno.036/Ge multiple quantum wells by quantum modelling
}

\author{
N Yahyaoui ${ }^{1}$, N Sfina ${ }^{1,4}$, J-L Lazzari ${ }^{2}$, A Bournel $^{3}$ and M Said ${ }^{1}$ \\ ${ }^{1}$ Laboratoire de la Matière Condensée et des Nanosciences (LMCN), Département de Physique, Faculté \\ des Sciences de Monastir, Avenue de l'Environnement, 5019 Monastir, Tunisia \\ ${ }^{2}$ Aix-Marseille Université, CNRS, CINaM UMR 7325, Campus de Luminy, Case 913, 13288 Marseille \\ cedex 9, France \\ ${ }^{3}$ Institut d'Electronique Fondamentale (IEF), UMR 8622 CNRS—Université Paris-Sud, Bât. 220, 91405 \\ Orsay cedex, France \\ E-mail: naima.yahyaoui@yahoo.fr and moncef_said@yahoo.fr
}

\begin{abstract}
$\mathrm{GeSn} / \mathrm{Ge}$ p-i-n photodetectors with practical $\mathrm{Ge}_{0.964} \mathrm{Sn}_{0.036}$ active layers are theoretically investigated. First, we calculated the electronic band parameters for the heterointerfaces between strained $\mathrm{Ge}_{1-x} \mathrm{Sn}_{x}$ and relaxed (001)-oriented Ge. The carrier transport in a p-i-n photodiode built on a ten-period $\mathrm{Ge}_{0.964} \mathrm{Sn}_{0.036} / \mathrm{Ge}$ multiple quantum well absorber was then analyzed and numerically simulated within the Tsu-Esaki formalism by self-consistently solving the Schrödinger and Poisson equations, coupled to the kinetic rate equations. Photodetection up to a $2.1 \mu \mathrm{m}$ cut-off wavelength is achieved. High responsivities of 0.62 $\mathrm{A} \mathrm{W}^{-1}$ and $0.71 \mathrm{~A} \mathrm{~W}^{-1}$ were obtained under a reverse bias voltage of $-3 \mathrm{~V}$ at peak wavelengths of $1550 \mathrm{~nm}$ and $1781 \mathrm{~nm}$, respectively. Even for this low Sn-fraction, it is found that the photodetector quantum efficiency (49\%@1.55 $\mu \mathrm{m}$ ) is higher than those of comparable pure-Ge devices at room temperature. Detectivity of $3.8 \times 10^{10} \mathrm{~cm} \mathrm{~Hz}^{1 / 2} \mathrm{~W}^{-1}$ and $7.9 \times 10^{10} \mathrm{~cm} \mathrm{~Hz}^{1 / 2}$ $\mathrm{W}^{-1}$ at $-1 \mathrm{~V}$ and $-0.5 \mathrm{~V}$, respectively, is achievable at room temperature for a $1550 \mathrm{~nm}$ wavelength peak of responsivity. This work represents a step forward in developing $\mathrm{GeSn} / \mathrm{Ge}$ based infrared photodetectors.
\end{abstract}

Keywords: infrared photodetectors, detectivity, band structure engineering

\section{Introduction}

In recent years, there has been increasing effort toward the development and realisation of group IV semiconductor optoelectronic devices, recently encouraged by their possible heterogeneous integration with CMOS technology on silicon

\footnotetext{
${ }^{4}$ Present address: Faculty of Science and Art KKU Mahail Assir, King Kaled University, Saudi Arabia.
}

on insulator (SOI) and germanium on insulator (GeOI) substrates. The progress made on key photonics components, including laser sources, optical modulators and photodetectors, has been demonstrated in detail [1]. Due to its relatively high absorption coefficient at $1.3-1.55 \mu \mathrm{m}, \mathrm{Ge}$ is regarded as the best candidate material for detection in this spectral range. However, the optical response of thin vertical Ge- based high frequency photodetectors at the telecommunication wavelength of $1.55 \mu \mathrm{m}$ is limited [2]. An 
Table 1. Summary of the lattice constants (in angstrom), elastic stiffness constants (in $10^{11} \mathrm{dyn} \mathrm{cm}^{-2}$ ), conduction and valence band deformation potentials, spin-orbit splitting and bulk bandgaps (in $\mathrm{eV}$ ) of $\mathrm{Ge}$ and $\alpha$-Sn. All symbols are used in their conventional meanings (see [19-21]).

\begin{tabular}{lllllllllll}
\hline & $\mathrm{a}$ & $\mathrm{c}_{11}$ & $\mathrm{c}_{12}$ & $\mathrm{a}_{\mathrm{c}}(\Gamma)$ & $\mathrm{a}_{\mathrm{c}}(\mathrm{L})$ & $E_{v, a v}$ & $\mathrm{~b}$ & $\Delta_{0}$ & $E_{g}(\Gamma)$ & $E_{g}(L)$ \\
\hline $\mathrm{Ge}$ & 5.6579 & 12.85 & 4.83 & -8.24 & -1.54 & 0 & -2.9 & 0.30 & 0.798 & 0.664 \\
$\alpha-\mathrm{Sn}$ & 6.4892 & 6.9 & 2.93 & -6 & -2.14 & 0.69 & -2.7 & 0.80 & -0.413 & 0.092 \\
\hline
\end{tabular}

increase of the responsivity for these wavelengths can be achieved by intermixing $\mathrm{Sn}$ to Ge [3]. Thus, $\mathrm{Ge}_{1-x} \mathrm{Sn}_{x}$ alloy is an interesting alternative since its bandgap, which is smaller than that of Ge, may further improve the optical response at $1.55 \mu \mathrm{m}$ and will push the spectral range to longer infrared wavelengths [4-6]. On the other hand, GeSn alloy has poor thermal stability [7, 8], which prevents its application in optoelectronic devices. In addition, the large lattice mismatch with $\mathrm{Ge}(\sim 15 \%)$ and the extremely low $(x<1 \%)$ solid solubility of $\mathrm{Sn}$ in Ge makes it very difficult to grow high-quality defect-free GeSn [9]. Notwithstanding, GeSn alloys with a few per cent Sn content can only be grown under non-equilibrium conditions. Despite these difficulties, some effort has been made toward the epitaxial growth of GeSn alloys [7, 8, 10] and the fabrication of GeSn based devices [4]. Recently, there have been reports on GeSn p-i-n photodetectors with small $\mathrm{Sn}$ content for full telecommunication spectral range applications [11]. These results show that adding $\mathrm{Sn}$ in a p-i-n Ge photodiode matrix also increases the responsivity of the detector in the telecom wavelength range and extends the cut-off wavelength beyond $1.7 \mu \mathrm{m}$ [12-14]. Su et al published the first GeSn p-i-n photodetector grown by solid-source molecular beam epitaxy with $3 \%$ Sn composition and $820 \mathrm{~nm}$ thick layers [14]. At $1640 \mathrm{~nm}$, they measured strong reverse voltage dependence with a small optical responsivity of $50 \mathrm{~mA} \mathrm{~W}^{-1}$ under zero bias operation. Strangely, the $\mathrm{Sn}$ incorporation shifts the detector cut-off responsivity towards the infrared spectral range by $60 \mathrm{~nm}$ only. The detection wavelength of GeSn p-i-n photodetectors fabricated on Ge substrate with $\mathrm{Sn}$ contents up to $3.6 \%$ in the active layer has recently reached $1.95 \mu \mathrm{m}$ [15]. Photodiodes built on lattice relaxed $\mathrm{Ge}_{1-x} \mathrm{Sn}_{x}$ layers $[15,16]$ may exhibit large densities of defects, such as dislocations acting as recombination traps, resulting in an excessive dark current. Strained $\mathrm{Ge}_{1-x} \mathrm{Sn}_{x} / \mathrm{Ge}$ multiple quantum wells (MQWs) may be used as an absorbing layer to adjust the absorption threshold and to reduce the dark current. However, owing to the serial resistance induced by the number of quantum barriers, a limited number of cells or coupled quantum wells in the superlattice regime should be used. In order to design such a device, we first determine the electronic properties of strained $\mathrm{Ge}_{1-x} \mathrm{Sn}_{x}$ on (001)-oriented Ge, such as bandgap energy and band discontinuities. Then, we propose a p-i-n infrared photodetector with an intrinsic absorbing layer formed by straincompensated $\mathrm{Ge} / \mathrm{Ge}_{0.964} \mathrm{Sn}_{0.036} / \mathrm{Ge}$ multi-quantum wells. This heterostructure is modeled with a system of Schrödinger and kinetic equations self-consistently solved with the Poisson equation. The current components due to these processes contributing to the total dark current are described. The room temperature performance is finally presented and discussed in terms of responsivity and detectivity.

\section{Material parameters}

The band offsets between binary $\alpha$-Sn containing alloys and Ge are not known experimentally [17]. In order to calculate the conduction and valence-band discontinuities between a $\mathrm{Ge}_{1-x} \mathrm{Sn}_{x}$ strained layer and (001)-oriented relaxed Ge substrate, we have adopted the calculation procedure of Van de Walle et al that we outlined in [18] using the parameters taken from [19-21] and the parameters listed in table 1. The following (1)-(4) approximated analytical laws of the conduction and valence-band discontinuities between strain split $L$ and $\Gamma$ valleys were extracted versus Sn composition:

$$
\begin{aligned}
& \Delta E_{\mathrm{C}}^{\Gamma}(x) \cong x(-1.543+2.054 x) \\
& \Delta E_{\mathrm{C}}{ }^{L}(x) \cong x(-0.249+0.853 x) \\
& \Delta E_{v, h h}(x) \cong x(1.424-0.169 x) \\
& \Delta E_{v, l h}(x) \cong x(1.121-0.159 x)
\end{aligned}
$$

with energies given in $\mathrm{eV} . \mathrm{Ge}_{1-x} \mathrm{Sn}_{x} / \mathrm{Ge}$ heterointerfaces have type I alignments at $\Gamma-\Gamma, L-L$ and $\Gamma-L$ respective critical points, meaning both electrons, heavy and light holes are confined into $\mathrm{Ge}_{x} \mathrm{Sn}_{x}$ alloys. The relaxed bandgaps of $\Gamma$ and $L$ valleys in the conduction band $(\mathrm{CB})$ relative to the top of the valence band (VB) are given by:

$$
E\left(\mathrm{Ge}_{1-x} \mathrm{Sn}_{x}\right)=(1-x) E_{\mathrm{Ge}}+x E_{\mathrm{Sn}}-c_{\mathrm{b}} x(1-x)
$$

where $c_{\mathrm{b}}$ is the bowing parameter for direct $\left(c_{\mathrm{b}}=2.87\right)$ or indirect $\left(c_{\mathrm{b}}=0.89\right)$ band gap energies. The values $E_{\mathrm{Ge}}$ and $E_{\mathrm{Sn}}$ of the bandgaps at $\Gamma$ and $L$ valleys for Ge and Sn are given in table 1 . The calculated bandgap energies $\left(E_{g}^{\Gamma}\right.$ and $E_{g}^{L}$, in eV) for strained $\mathrm{Ge}_{1-x} \mathrm{Sn}_{x}$ layers are given by the analytical expressions below:

$$
\begin{aligned}
& E_{g}^{(\Gamma-h h)}(x)=0.798-2.967 x+2.224 x^{2} \\
& E_{g}^{(I-l h)}(x)=0.798-2.660 x+2.214 x^{2} \\
& E_{g}^{(L-h h)}(x)=0.659-1.673 x+1.022 x^{2} \\
& E_{g}^{(L-l h)}(x)=0.659-1.369 x+1.012 x^{2} .
\end{aligned}
$$

The resulting plots of the energy gaps for compressively strained and relaxed alloys including experimental data values are summarized in figure 1. It is found that pseudomorphic $\mathrm{Ge}_{1-x} \mathrm{Sn}_{x} / \mathrm{Ge}(001)$ alloys may have a direct bandgap at $\mathrm{Sn}$ fractions higher than $10.5 \%$ at room temperature. Regarding 


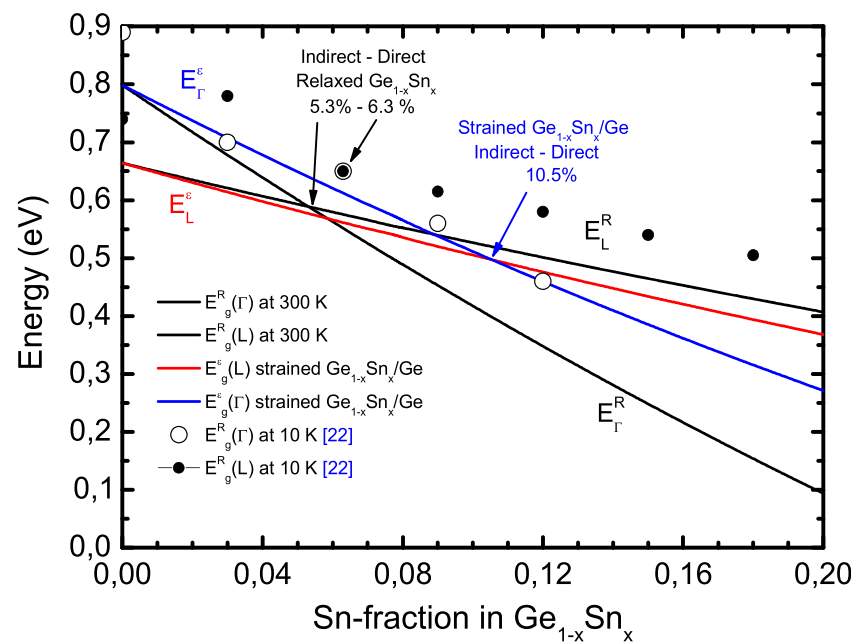

Figure 1. Energy gaps of pseudomorphic and relaxed $\mathrm{Ge}_{1-x} \mathrm{Sn}_{x}$ alloys. The dashed red and black lines display the indirect pseudomorphic $E_{L}^{\varepsilon}$ and relaxed $E_{L}^{R}$ bandgaps, respectively; solid blue and black lines are the direct pseudomorphic $E_{\Gamma}^{\varepsilon}$ and relaxed $E_{\Gamma}^{R}$ bandgaps, respectively. Symbols correspond to available experimental data [22] and $E_{\Gamma}^{R}$ and $E_{L}^{R}$ are obtained by the empirical pseudo-potential method (EPM)

the unstrained $\mathrm{Ge}_{1-x} \mathrm{Sn}_{x}$ alloys, indirect-to-direct crossing occurs at a $\mathrm{Sn}$ fraction of $5.3 \%$ at room temperature. The latter values are consistent with the experimental crossovers found in [22] at about 17 and $6.3 \%$ respectively for temperatures around $10 \mathrm{~K}$. Additional experimental evidence of the indirect -direct bandgap transition for pseudomorphic $\mathrm{Ge}_{1-x} \mathrm{Sn}_{x} / \mathrm{Ge}$ (001) would be of fundamental and practical importance.

\section{Studied structure and modeling}

The structure under investigation consists of 10 periods of intrinsic $\mathrm{Ge}_{0.964} \mathrm{Sn}_{0.036} / \mathrm{Ge} \mathrm{QW}$ cell. Both compressively strained $\mathrm{Ge}_{0.964} \mathrm{Sn}_{0.036}$ and relaxed Ge barriers are $10 \mathrm{~nm}$ thick. The device is sketched in figure 2 . The material parameters used are taken from [23-25] and summarized in table 2. The compressive lattice mismatch between $\mathrm{Ge}_{0.964} \mathrm{Sn}_{0.036}$ and $\mathrm{Ge}$ is $0.52 \%$ while the average mismatch of the $200 \mathrm{~nm}$-thick stacks is $0.26 \%$. Thus the chosen thicknesses are reasonable values regarding the critical thickness of $\mathrm{Ge}_{0.964} \mathrm{Sn}_{0.036}$ layers and strain accumulation within the stack. However, thicker MQWs for better absorption would imply strain compensation through the use of $\mathrm{Si}_{0.13} \mathrm{Ge}_{0.87}$ barriers under tensile strain. The active region is embedded between $70 \mathrm{~nm}$-thick $\mathrm{n}$ and p-doped Ge contact layers doped at $N_{\mathrm{A}}=N_{\mathrm{D}}=1 \times 10^{18} \mathrm{~cm}^{-3}$. For the sake of simplicity, we assume that there are no intrinsic impurities or defects in the host lattice.

The theoretical model and numerical methods used in this paper are based on the self-consistent solving of the onedimensional Schrödinger equation in the effective mass approximation [26-29] and Poisson equations for electrons and holes. Numerically, the problem was treated using the finite differential method. The electron and hole densities $n(z)$ and $p(z)$ in the contact regions are calculated according to the Boltzmann statistic. Figure 3 shows the conduction- and

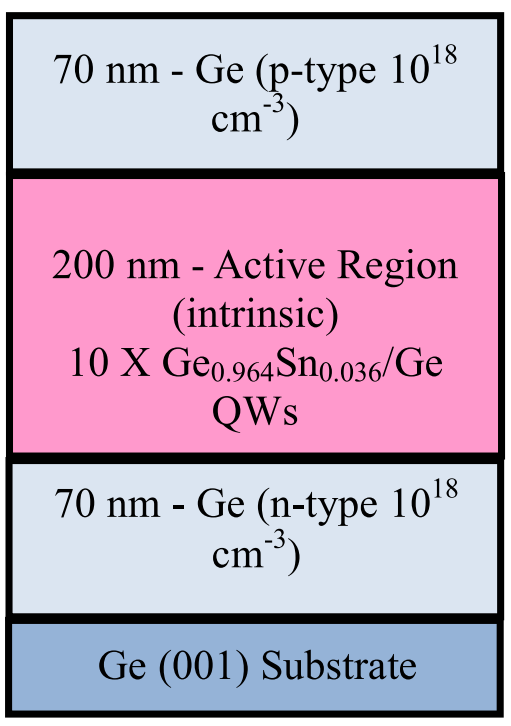

Figure 2. Schematic diagram of a p-i-n photodetector. The crosssectional view shows the structure of strained GeSn/Ge multiple quantum wells grown on germanium substrate.

valence-band profiles for this ten-period $\mathrm{Ge}_{0.964} \mathrm{Sn}_{0.036} / \mathrm{Ge}$ MQW at zero bias. The ground level is drawn for electron, light-hole and heavy-hole with the associated wave function. Electrons fill all available states in the active part of the device according to the Fermi-Dirac $f(E)$ distribution. The Fermi level is linearly varying from $E_{\mathrm{F}(p)}=E_{\mathrm{F}}$ at the $p$ contact to $E_{\mathrm{F}(n)}=E_{\mathrm{F}}-q V_{\text {bias }}$ at the $n$ contact, where $V_{\text {bias }}$ is the applied bias. The formula after Tsu and Esaki generalized for the case of several conduction valleys and anisotropy of effective mass is then used for calculation of the tunneling current. For parabolic dispersion relations with integration over the transverse mass component only, the current density from extended and localized states is written as [30]:

$$
\begin{aligned}
J(V)= & \frac{q m^{*} k_{\mathrm{B}} T}{2 \pi^{2} \hbar^{3}} \\
& \times \int_{0}^{\infty} \operatorname{tr}\left(E_{z}, V_{\text {bias }}\right) L n \\
& \times\left[\frac{1+\exp \left(E_{f}-E_{z} / k_{\mathrm{B}} T\right)}{1+\exp \left(E_{f}-E_{z}-q V_{\text {bias }} / k_{\mathrm{B}} T\right)}\right] \mathrm{d} E_{z}
\end{aligned}
$$

where the transmission coefficient $\operatorname{tr}\left(E_{Z}\right)$ is deduced from the transfer matrix formalism within the step approximation as for the potential [31]; $T$ is the absolute temperature and $k_{\mathrm{B}}$ and $\hbar$ are the Boltzmann and reduced Planck constants, respectively. For an analysis of the thermally activated carrier transport, the current density can be described using the rates $g_{i \rightarrow i+1}, \quad g_{i+1 \rightarrow i}$ of electron (or hole) transfer components in two opposite directions, according to [32]:

$$
\begin{aligned}
J= & \frac{\partial n_{i}}{\partial t}+\frac{\partial p_{i}}{\partial t} \\
= & \left(g_{i-1 \rightarrow i}^{n} n_{i-1}-n_{i} g_{i \rightarrow i+1}^{n}-n_{i} g_{i \rightarrow i-1}^{n}+n_{i+1} g_{i+1 \rightarrow i}^{n}\right) \\
& +\left(-g_{i-1 \rightarrow i}^{p} p_{i-1}+p_{i} g_{i \rightarrow i+1}^{p}\right. \\
& \left.+p_{i} g_{i \rightarrow i-1}^{p}-p_{i+1} g_{i+1 \rightarrow i}^{p}\right)
\end{aligned}
$$




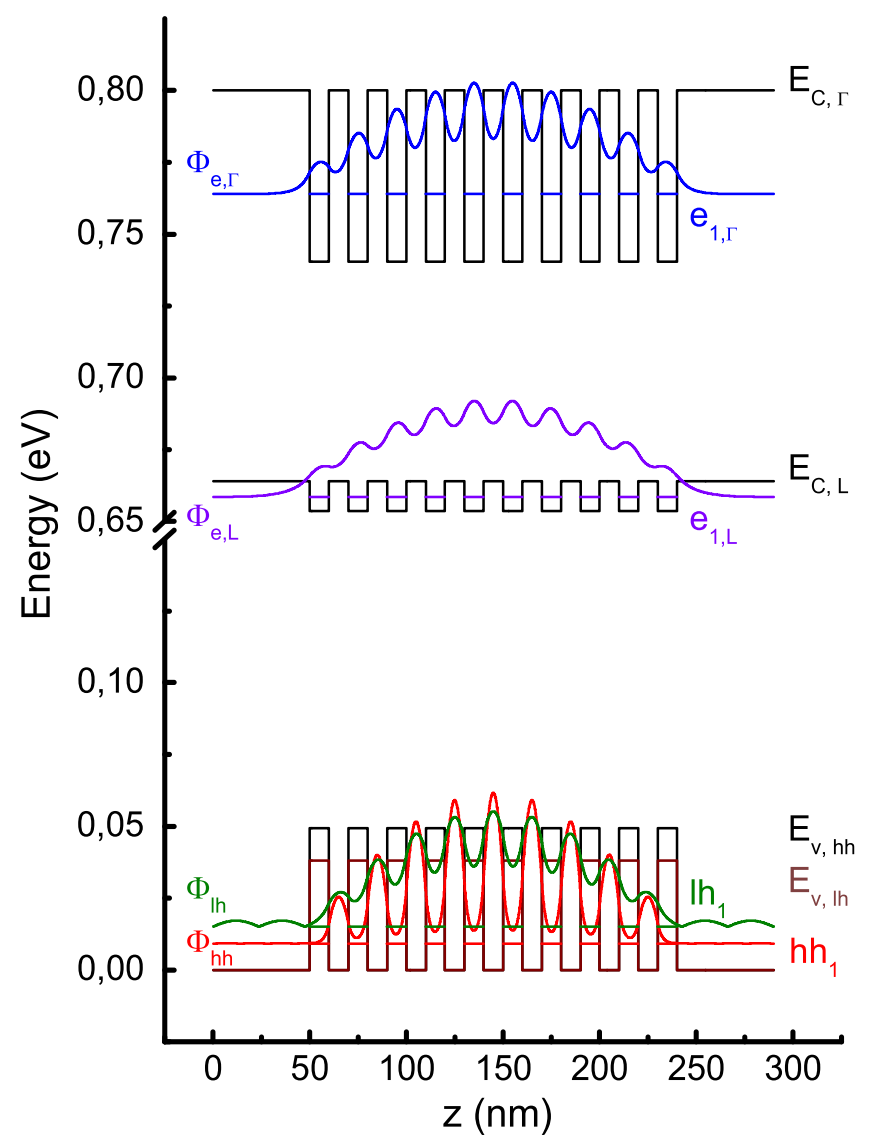

Figure 3. Conduction- and valence-band profiles for ten-period $\mathrm{Ge}_{0.964} \mathrm{Sn}_{0.036} / \mathrm{Ge}$ MQWs (the values for band offset and bandgap of $\mathrm{Ge}_{0.964} \mathrm{Sn}_{0.036} / \mathrm{Ge}$ are summarized in table 2). The fundamental electron, light-hole and heavy-hole levels are drawn with their relative wave function at $\Gamma$ and $L$ valleys. Note the envelopes of electron and hole wave functions, meaning $\mathrm{Ge}_{0.964} \mathrm{Sn}_{0.036}$ quantum wells are coupled with a $10 \mathrm{~nm}$ thick Ge spacer.

where $n_{i}\left(p_{i}\right)$ is the electron (hole) concentration in the $i$ th mesh point. This rate, defined within the thermally activated transport theory, includes the image-force effect in the following way [33]:

$$
\begin{aligned}
g_{i \rightarrow i+1}^{n, p}= & \omega_{0 i}^{n, p} \exp \left(\frac{q\left(\phi_{i}-\phi_{i+1}\right)}{k_{\mathrm{B}} T}\right) \\
& \times \exp \left(-\left(\Delta E_{i}^{C, V}-\sqrt{\frac{q^{3}\left(\phi_{i}-\phi_{i+1}\right)}{16 \pi \varepsilon_{i} \varepsilon_{0} h z_{i}}}\right) / k_{\mathrm{B}} T\right)
\end{aligned}
$$

where $h z_{i}$ is the step of the mesh, $\phi_{i}$ is the electrostatic potential in the $i$ th mesh (obtained by solving the Poisson equation), $\varepsilon_{i}$ is the dielectric constant in the $i$ th mesh, and $\omega_{0}$ is the frequency of the local carrier oscillations along the multiple quantum well. The system of kinetic equations has been solved for the stationary case.

In the absence of the optical excitation $G_{n i}=G_{p i}=0$, it is worth comparing the generalized formula of the dark current density described by equations (10)-(12) with the classical expression given by continuity equations [27] to account for additional current contributions:

$$
\begin{aligned}
J= & \frac{\partial n_{i}}{\partial t}+\frac{\partial p_{i}}{\partial t} \\
= & G_{n i}+G_{p i} \\
& -\left(\frac{n_{i}-n_{0}}{\tau_{n, i}^{\mathrm{R}, \mathrm{NR}}} \frac{p_{0}+n_{i}}{p_{0}}-\frac{p_{i}-p_{0}}{\tau_{p, i}^{\mathrm{R}, \mathrm{NR}}} \frac{n_{0}+p_{i}}{n_{0}}\right) \\
& +J_{\text {Tunnel }}^{\text {Band to Band }}+J_{\text {Tunnel }}^{\text {Electrons }}+J_{\text {Tunnel }}^{\text {Holes }} \\
& +\left(n_{i} \mu_{n i} \frac{\partial \xi_{i}}{\partial z_{i}}+\mu_{n i} \xi_{i} \frac{\partial n_{i}}{\partial z_{i}}\right. \\
& \left.-p_{i} \mu_{p i} \frac{\partial \xi_{i}}{\partial z_{i}}-\mu_{p i} \xi_{i} \frac{\partial p_{i}}{\partial z_{i}}\right) \\
& +\left(D_{i}^{n} \frac{\partial^{2} n_{i}}{\partial z_{i}^{2}}+D_{i}^{p} \frac{\partial^{2} p_{i}}{\partial z_{i}^{2}}\right)
\end{aligned}
$$

where $n_{\mathrm{o}}$ and $p_{\mathrm{o}}$ are the electron and hole free carrier concentrations at thermodynamic equilibrium, $G_{n i}$ and $G_{p i}$ are the electron and hole optical generation rates, $\mu_{i}{ }^{n, p}$ is the electron (or hole) mobility, $D_{i}^{n, p}(T)=\frac{k_{\mathrm{B}} T}{q} \mu_{i}^{n, p}(T)=\frac{k_{\mathrm{B}} T}{q}\left(\frac{q \tau_{i}^{n, p}}{m_{e, h}^{\perp}}\right)$ is the electron (or hole) diffusion coefficient, $\tau_{i}^{n, p}$ is the averaged scattering times for electrons or holes, $\xi_{i}=\partial \phi_{i} / \partial z_{i}$ is the applied electric field, and $\tau_{n, p i}^{\mathrm{R}, \mathrm{NR}}$ is the average time for the radiative and non radiative recombination process for electrons (or holes) in the $i$ th point mesh, e.g. $\frac{1}{\tau_{n, p i}^{\mathrm{R} N \mathrm{NR}}}=\frac{1}{\tau_{n, p i}^{\mathrm{R}}}+\frac{1}{\tau_{n, p i}^{\mathrm{NR}}}$. In the present work, we do not consider the contributions on the generation-recombination current of the Shockley-Read-Hall recombination of carriers on traps, e.g. $\tau_{n, p i}^{\mathrm{NR}} \rightarrow \infty$ for first term of equation (13), neither the three carrier Auger mechanisms, nor the band-to-band tunneling mechanism that are valid assumptions for low concentrations of photo-carriers and low reverse voltages of a p-i-n photodiode. The two sets of equations (10)-(12) or (13) thus account for same current contributions.

In the particular case of a GeSn absorbing layer (not MQWs), e.g. $\Delta E_{i}^{C, V}=0$ and no image-force effect, if one considers a first-order finite development on the electrostatic potential difference within a mesh, the transfer rate given in equation (12) reduces to: $g_{i \rightarrow i+1}^{n, p} \cong \omega_{0 i}^{n, p}\left(1+\frac{q\left(\phi_{i}-\phi_{i+1}\right)}{k_{\mathrm{B}} T}\right)$. Using the same linear development for all derivative terms of the conduction and diffusion currents given in second part of equation (13), by comparing with those extracted from equation (13), one can find [34]:

$$
\begin{aligned}
\omega_{0 i}^{n, p} & \cong \frac{D_{i}^{n, p}(T)}{\left(h z_{i}\right)^{2}}=\frac{k_{\mathrm{B}} T}{q} \frac{\mu_{i}^{n, p}(T)}{\left(h z_{i}\right)^{2}} \\
& =\frac{k_{\mathrm{B}} T}{q}\left(\frac{q \tau_{i}^{n, p}}{m_{e, h}^{\perp}}\right) \frac{1}{\left(h z_{i}\right)^{2}} .
\end{aligned}
$$

In the absence of tunnel barriers, this remarkable formula links the frequency of local carrier oscillations along the structure and their averaged times $\tau_{i}^{n, p}$ of scattering with impurities, lattice phonons and alloy disorder. It is valid only if the step of the mesh $h z_{i}$ is of the order of magnitude 
Table 2. Parameters at $300 \mathrm{~K}$ used for the simulation of the p-i-n $\mathrm{Ge}_{0.964} \mathrm{Sn}_{0.036} / \mathrm{Ge} \mathrm{MQW}$ based photodetectors.

\begin{tabular}{ll}
\hline Parameters & Values \\
\hline Electron effective mass $\mathrm{Ge}_{0.964} \mathrm{Sn}_{0.036} / \mathrm{Ge} m_{\Gamma, e}^{*}$ & $0.032 \mathrm{~m}_{0}[23]$ \\
Electron effective mass $\mathrm{Ge}_{0.964} \mathrm{Sn}_{0.036} / \mathrm{Ge} m_{L, e}^{*}$ & $0.078 \mathrm{~m}_{0}[23]$ \\
Heavy hole effective mass $\mathrm{Ge}_{0.964} \mathrm{Sn}_{0.036} / \mathrm{Ge} m_{h h}^{*}$ & $0.199 \mathrm{~m}_{0}[23]$ \\
Light hole effective mass $\mathrm{Ge}_{0.964} \mathrm{Sn}_{0.036} / \mathrm{Ge} m_{l h,}^{*}$ & $0.061 \mathrm{~m}_{0}[23]$ \\
Bandgap $\mathrm{Ge}_{0.964} \mathrm{Sn}_{0.036} / \mathrm{Ge} E_{g}^{\Gamma-h h}$ & $0.694 \mathrm{eV}$ (present work) \\
Bandgap $\mathrm{Ge}_{0.964} \mathrm{Sn}_{0.036} / \mathrm{Ge} E_{g}^{\Gamma-l h}$ & $0.705 \mathrm{eV}$ (present work) \\
Bandgap $\mathrm{Ge}_{0.964} \mathrm{Sn}_{0.036} / \mathrm{Ge} E_{g}^{L-h h}$ & $0.600 \mathrm{eV}$ (present work) \\
Bandgap $\mathrm{Ge}_{0.964} \mathrm{Sn}_{0.036} / \mathrm{Ge} E_{g}^{L-l h}$ & $0.611 \mathrm{eV}$ (present work) \\
Conduction band-offset $\mathrm{Ge}_{0.964} \mathrm{Sn}_{0.036} / \mathrm{Ge} \Delta E_{\Gamma}^{C}$ & $-0.053 \mathrm{eV}$ (present work) \\
Conduction band-offset $\mathrm{Ge}_{0.964} \mathrm{Sn}_{0.036} / \mathrm{Ge} \Delta E_{L}^{C}$ & $-0.008 \mathrm{eV}$ (present work) \\
Valence band-offset $\mathrm{Ge}_{0.964} \mathrm{Sn}_{0.036} / \mathrm{Ge} \Delta E_{h h}^{V}$ & $0.051 \mathrm{eV}$ (present work) \\
Valence band-offset $\mathrm{Ge}_{0.964} \mathrm{Sn}_{0.036} / \mathrm{Ge} \Delta E_{l h}^{V}$ & $0.040 \mathrm{eV}$ (present work) \\
Electron carrier recombination lifetime $\tau_{n i}$ & $9 \mu \mathrm{s} \mathrm{[24]}$ \\
Electron carrier recombination lifetime $\tau_{p i}$ & $9 \mu \mathrm{s} \mathrm{[24]}$ \\
Electron carrier mobility $\mu_{n i}(T)$ & $\approx\left(m_{i, e}^{\perp}\right)^{-1 / 2} T^{3 / 2}$ [25] \\
Hole carrier mobility $\mu_{p i}(T)$ & $\approx\left(m_{i, h}^{\perp}\right)^{-1 / 2} T^{3 / 2}$ [25] \\
Acceptor concentration, $N_{\mathrm{A}}$ & $10^{18} \mathrm{~cm}^{-3}$ \\
Donor concentration, $N_{\mathrm{D}}$ & $10^{18} \mathrm{~cm}{ }^{-3}$ \\
\hline
\end{tabular}

of the QW width. For a $10 \mathrm{~nm}$ thick Ge QW, taking a room temperature mobility of $3900 \mathrm{~cm}^{2} \mathrm{~V}^{-1} \mathrm{~s}^{-1}$ and $1900 \mathrm{~cm}^{2} \mathrm{~V}^{-1} \mathrm{~s}^{-1}$ for electrons and holes, one can find $\omega_{0}^{n, p} \cong(10-15 f s)^{-1}$, respectively.

\section{Results and discussion}

Figure 4 compares the dark current-voltage $(I-V)$ characteristics calculated at room temperature for bulk $\mathrm{Ge}_{0.964} \mathrm{Sn}_{0.036}$ and $\mathrm{Ge}_{0.964} \mathrm{Sn}_{0.036} / \mathrm{Ge} \mathrm{MQW}$ active regions. A good agreement of the calculated and experimental dark currents [15] is obtained for a $200 \mu \mathrm{m}$ thick bulk $\mathrm{Ge}_{0.964} \mathrm{Sn}_{0.036}$ active region, a mesa diode diameter of $150 \mu \mathrm{m}$, and a $9 \mu \mathrm{s}$ [24] generation -recombination time of carriers. Such an experimental device has a typical dark-current of $1.210^{-6} \mathrm{~A}$ at $-1 \mathrm{~V}$. The use of MQWs as the active region increases the serial resistance [22] and thus decreases the dark current around 3.1 $10^{-7} \mathrm{~A}$ for the same $-1 \mathrm{~V}$ reverse voltage.

In order to understand the evolution of the dark current with the bias voltage, it is necessary to determine the respective influence of each component originating from the different considered mechanisms that include the conduction, diffusion, recombination and thermally activated tunnel currents. The calculation of each current component has been carried out at room temperature using the approach described in the previous section. Results are illustrated in figure 5. It is shown that the thermal diffusion and the well describe the forward bias characteristic while the behavior of the total dark current under reverse polarization thoroughly agree with the sum of the tunneling and recombination current contributions. The diffusion component remains the main mechanism for low $(<0.5 \mathrm{~V})$ direct bias voltages.

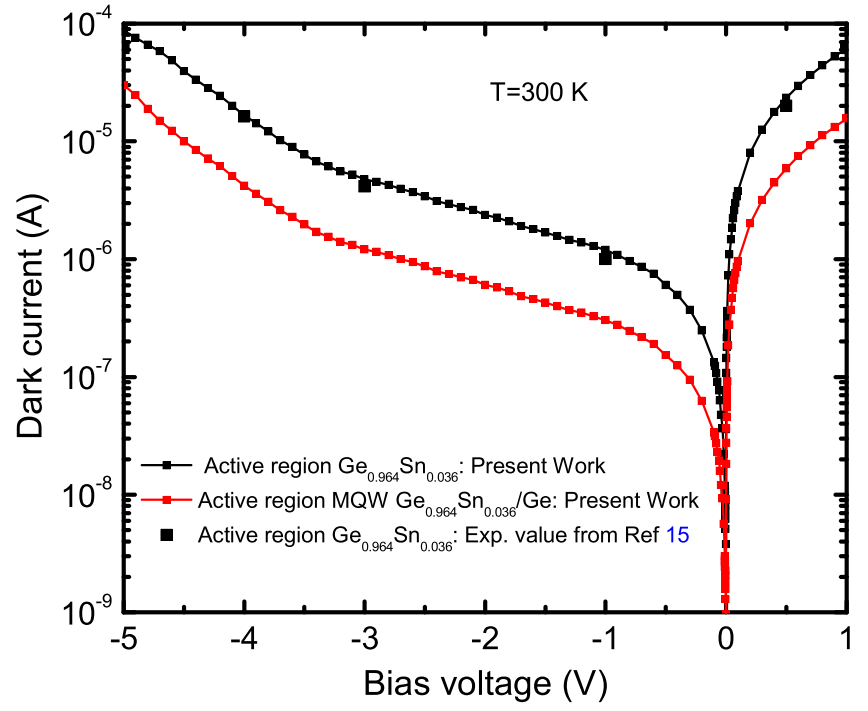

Figure 4. Dark current-voltage characteristics of a GeSn p-i-n photodetector with a Sn content of $3.6 \%$ for devices with different active region $\mathrm{Ge}_{0.964} \mathrm{Sn}_{0.036}$ and $\mathrm{MQW} \mathrm{Ge} / \mathrm{Ge}_{0.964} \mathrm{Sn}_{0.036} / \mathrm{Ge}$. The symbols are the experimental data taken from [15].

Next we have investigated the influence of $\alpha$-Sn concentration on the $I-V$ dark characteristics. The results of our simulations of the dark current-voltage characteristics at room temperature for a pure bulk Ge $\mathrm{p}$-i-n photodiode and two devices based on ten-period strained $\mathrm{Ge}_{1-\mathrm{x}} \mathrm{Sn}_{\mathrm{x}} / \mathrm{Ge}$ MQWs with different $\alpha$-Sn compositions, are illustrated in figure 6.

The dark current density not only indicates the material quality but also determines the optical receiver sensitivity. A direct comparison with the experimental results pointed out by recent investigations [15] proves that our proposed 


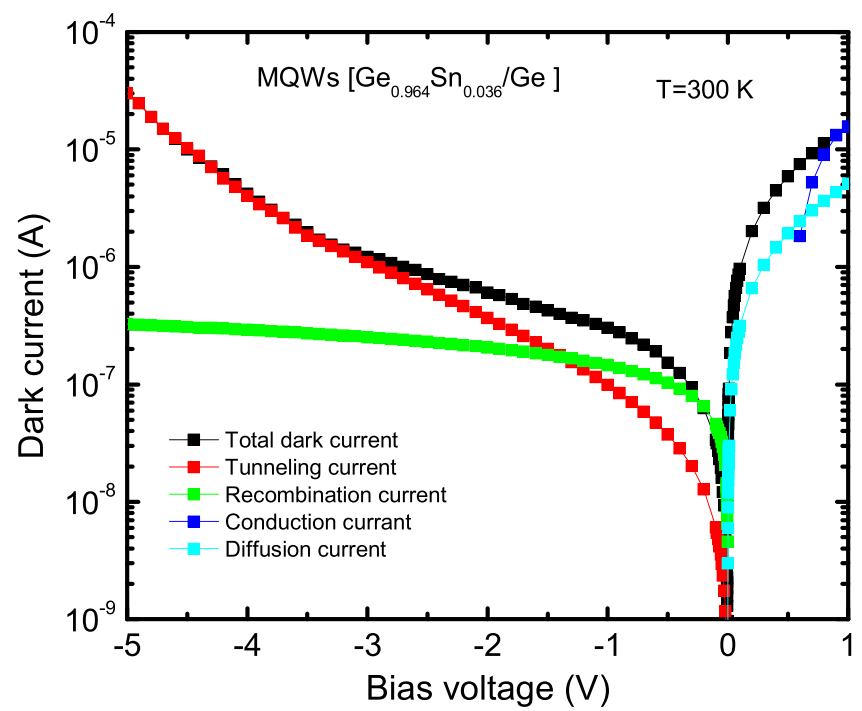

Figure 5. Total dark current at $300 \mathrm{~K}$ as a function of bias voltage from $-5 \mathrm{~V}$ to $1 \mathrm{~V}$ is shown with four distinct regimes and different dominant mechanisms, the conduction current density, diffusion current density, recombination current density and tunneling current density.

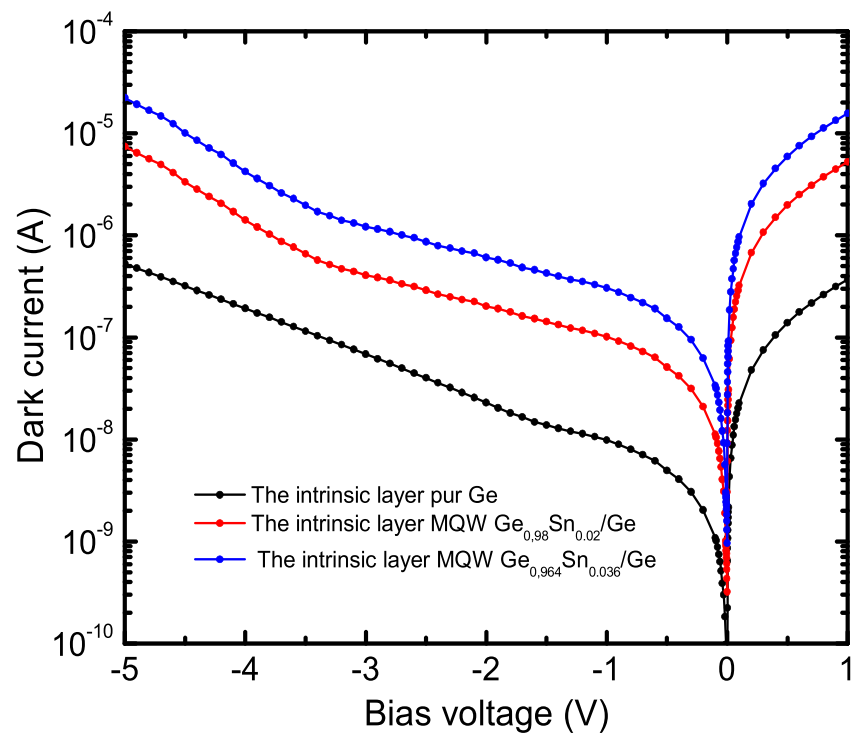

Figure 6. $I-V$ characteristics of the devices with different $\mathrm{Sn}$ concentrations (device radius $150 \mu \mathrm{m}$ ).

structure allows improvement of the performance of the photodiode. The optical responsivity is obtained using the following expression:

$$
\begin{aligned}
R(\lambda)= & \frac{\eta_{i} q \lambda \Omega}{h c} \frac{\Gamma \alpha(\lambda, V)}{\Gamma \alpha(\lambda, V)+\alpha_{i}} \\
& \times\left(1-\exp \left(-\left(\alpha_{i}+\Gamma \alpha(\lambda, V)\right) L\right)\right.
\end{aligned}
$$

where $\eta_{i}$ is the internal quantum efficiency, $\Gamma$ is the confinement factor of the optical mode within the multiple quantum wells, $\alpha(\lambda, V)$ is the absorption coefficient at wavelength $\lambda$ and bias $V, L$ is the waveguide length, and $\Omega$ is the coupling efficiency, including mode size mismatch and reflection [35]. $\alpha_{i}$ accounts for all the propagation losses that do not generate photocurrent. We used $\eta_{i}=1, \Gamma=0.38$, $\Omega=0.25, \alpha_{i}=120 \mathrm{~cm}^{-1}$ and $\alpha(\lambda, V)$ was theoretically calculated according to the formula outlined in our previous work [23].

The room temperature responsivity for three reverse bias voltages $(0 \mathrm{~V},-1 \mathrm{~V}$ and $-3 \mathrm{~V})$ are given in figure $7(\mathrm{a})$. This responsivity versus wavelength $(0.9-2.3 \mu \mathrm{m})$ shows two peaks. These peaks corresponding to the $\mathrm{hh}_{1}-\mathrm{c} \Gamma_{1}$ transition energy and the strained direct bandgap of $\mathrm{Ge}_{0.964} \mathrm{Sn}_{0.036} / \mathrm{Ge}$ QWs are observed around $1550 \mathrm{~nm}$ and $1781 \mathrm{~nm}$, respectively. Similarly, we can see that the responsivity decreases when the reverse bias voltage increases. Under a reverse bias voltage of $-3 \mathrm{~V}$, the responsivities of $0.62 \mathrm{~A} \mathrm{~W}^{-1}$ at $1550 \mathrm{~nm}$ and $0.71 \mathrm{~A} \mathrm{~W}^{-1}$ at $1781 \mathrm{~nm}$ are obtained at room temperature.

Afterwards, we investigated the effect of the concentration of $\alpha$-Sn on the optical responsivity for the $\mathrm{Ge} / \mathrm{Ge}_{1-x} \mathrm{Sn}_{x} /$ Ge p-i-n photodetector at zero bias. Figure 7(b) shows that an increase of the optical responsivity for higher wavelengths can be observed with increasing $\alpha$-Sn content at $0 \mathrm{~V}$. We found that the $\mathrm{Ge}_{0.98} \mathrm{Sn}_{0.02} / \mathrm{Ge} \mathrm{MQW}$ photodetector is achieved with a responsivity of $240 \mathrm{~mA} \mathrm{~W}^{-1}$ at the telecommunication wavelength of $1.55 \mu \mathrm{m}$. The response of the photodetector with $\mathrm{Sn} 2 \%$ is significantly higher than that reported in [36]. In addition, a rise of $3.6 \%$ of the $\alpha$-Sn amount leads to an increase greater than an order of magnitude on the responsivity. Indeed, the optical responsivity curve of the $\mathrm{Ge}_{0.964} \mathrm{Sn}_{0.036} / \mathrm{Ge}$ p-i-n MQW is shifted $\sim 500 \mathrm{~nm}$ to higher wavelengths compared with pure Ge. At $\lambda=1.781 \mu \mathrm{m}$, an optical responsivity of $\sim 306 \mathrm{~mA} \mathrm{~W}^{-1}$ is observed for the $\mathrm{Ge}_{1-x} \mathrm{Sn}_{x} / \mathrm{Ge}$ p-i-n photodetector with 3.6\% $\alpha$-Sn, whereas the Ge reference photodetector without $\alpha$-Sn shows an optical responsivity of $0.17 \mathrm{~A} \mathrm{~W}^{-1}$. Compared to $\mathrm{Ge}$ photodetector, responsivity is improved in the whole wavelength range from $0.9 \mu \mathrm{m}$ to $2.3 \mu \mathrm{m}$. Gassenq et al [37] found that an increase of $S_{n}$ content enhances the responsivity of the detector in the telecom wavelength range and extends the cut-off wavelength beyond $2.4 \mu \mathrm{m}$.

Besides the dark current, we can test the MQW's performance by its detectivity $D^{*}$ which depends on the wavelength of incident light $\lambda$. The detectivity is given by the B L Sharma simplified formula:

$$
D^{*}(\lambda)=\frac{1}{2} R(\lambda)\left(q J_{0}\right)^{-\frac{1}{2}}
$$

where $\lambda$ is the operating wavelength and $J_{0}$ is the dark current density.

Using this expression in the region of zero bias voltage of the $\mathrm{p}-\mathrm{n}$ junction, we have calculated room-temperature specific detectivity at a $1550 \mathrm{~nm}$ wavelength peak of responsivity. $D^{*} 7.910^{10} \mathrm{~cm} \mathrm{~Hz}^{1 / 2} \mathrm{~W}^{-1}$ and $\sim 3.810^{10} \mathrm{~cm} \mathrm{~Hz}^{1 / 2} \mathrm{~W}^{-1}$ have been achieved under reverse bias voltage of $-0.5 \mathrm{~V}$ and $-1 \mathrm{~V}$, respectively, at $300 \mathrm{~K}$. We have not investigated the temperature effect. Conley et al [38] found that the detectivity of $\mathrm{GeSn}$ photoconductors as expected increases with Sn increasing content when temperature decreases. But their device exhibits a poor value of detectivity of about $1109 \mathrm{~cm}$ 

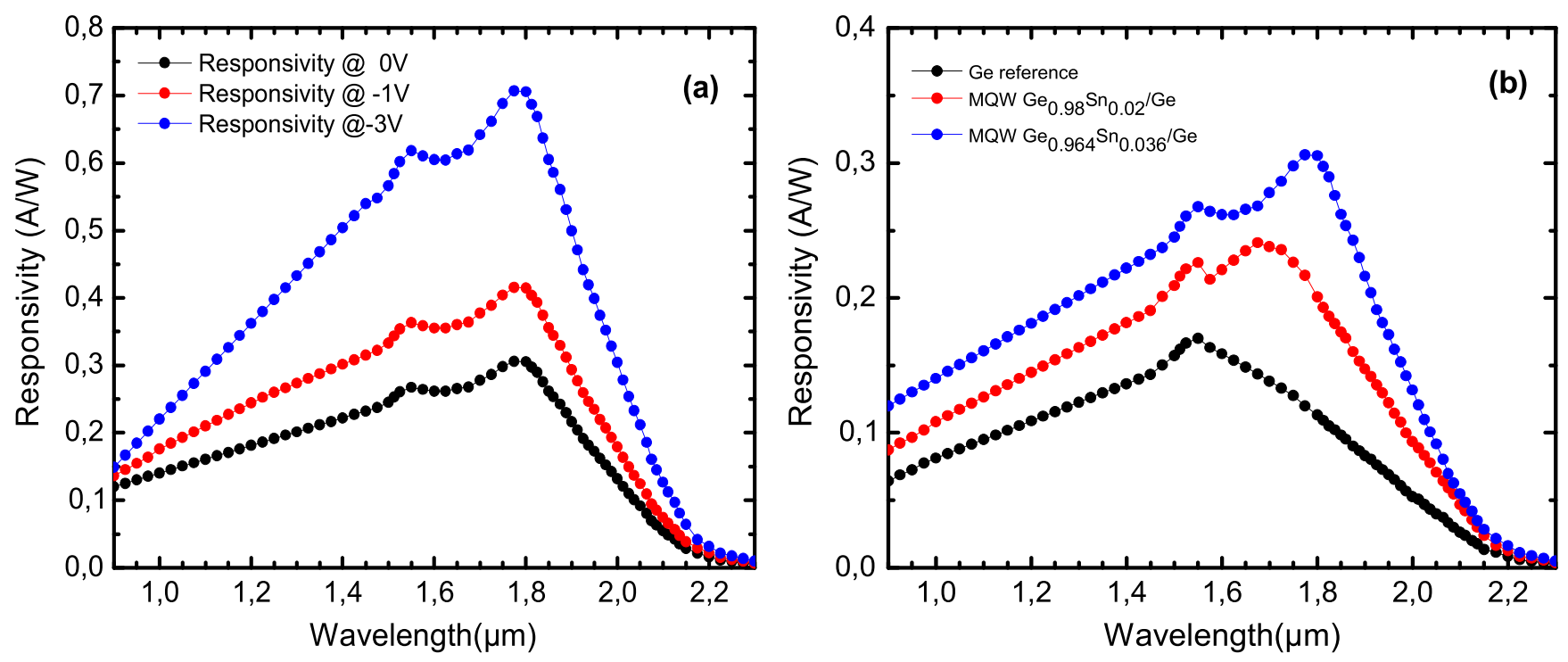

Figure 7. (a) Optical responsivity from $\lambda=900 \mathrm{~nm}$ up to $\lambda=2300 \mathrm{~nm}$ for the $\mathrm{Ge}_{0.964} \mathrm{Sn}_{0.036} / \mathrm{Ge}$ p-i-n photodetectors at $0,-1$ and $-3 \mathrm{~V}$, respectively. (b) Responsivity versus wavelength with different Sn concentrations at reverse zero bias.

$\mathrm{Hz}^{1 / 2} \mathrm{~W}^{-1}$ at $1.55 \mu \mathrm{m}$, as compared to the results of Zhang et al [15].

$\mathrm{GeSn} / \mathrm{Ge} \mathrm{p}-\mathrm{i}-\mathrm{n}$ photodetectors operating in the infrared region $(1.3-1.55 \mu \mathrm{m})$ are finding extensive applications in long haul and high bit rate optical communication systems and local area networks [39-41].

In addition to optical communication, these devices are also useful for sensing applications as they have superior electro-optical characteristics, namely low dark current, high quantum efficiency, greater sensitivity and high speed of response.

\section{Conclusion}

In summary, we have theoretically studied the group-IV semiconductor in which the GeSn relaxed alloys have a direct bandgap for $5.3 \% \alpha$-Sn. Our investigation predicts, at room temperature, a direct bandgap for strained $\mathrm{Ge}_{1-x} \mathrm{Sn}_{x} / \mathrm{Ge}(001)$ structures at a $\alpha$-Sn fraction higher than $10.5 \%$. We have theoretically simulated the characteristics of GeSn/Ge p-i-n photodetector with Sn contents up to $3.6 \%$. The electrical and optical properties are compared with a similar Ge p-i-n reference photodetector without Sn. An increase of Sn content shifts the optical responsivity curve to higher wavelengths compared with pure Ge. The simulation of the responsivity $(0.9-2.3 \mu \mathrm{m})$ shows that the detectors have a photo-response up to $2.1 \mu \mathrm{m}$, covering the entire telecommunication range. In particular, considering the absorption of the strained direct bandgap of the $\mathrm{Ge}_{0.964} \mathrm{Sn}_{0.036} / \mathrm{Ge}$ quantum wells, good responsivities of $0.62 \mathrm{~A} \mathrm{~W}^{-1}$ at $1550 \mathrm{~nm}$ and $0.71 \mathrm{~A} \mathrm{~W}^{-1}$ at $1781 \mathrm{~nm}$ were obtained at a reverse bias voltage of $-3 \mathrm{~V}$. Consequently, $\mathrm{Ge}_{1-x} \mathrm{Sn}_{x} / \mathrm{Ge}$ heterostructures are a promising candidate material not only for all telecommunication bands in optoelectronics but also for near infrared applications.

\section{References}

[1] Reed G T 2008 Silicon Photonics: The State of the Art (New York: Wiley)

[2] Wang J and Lee S 2011 Sensors 11696

[3] Soref R 2006 J. Sel. Top. Quantum Electron. 121678

[4] Mathews J, Roucka R, Xie J, Yu S Q, Menendez J and Kouvetakis J 2009 Appl. Phys. Lett. 95133506

[5] Werner J, Oehme M, Schmid M, Kaschel M, Schirmer A, Kasper E and Schulze J 2011 Appl. Phys. Lett. 98061108

[6] Coppinger M, Hart J, Bhargava N, Kim S and Kolodzey J 2013 Appl. Phys. Lett. 102141101

[7] He G and Atwater H A 1996 Appl. Phys. Lett. 68664

[8] Gurdal O, Desjardins P, Carlsson J R A, Taylor N, Radamson H H, Sundgren J-E and Greene J E 1998 J. Appl. Phys. 83162

[9] Grzybowski G, Beeler R T, Jiang L, Smith D J, Kouvetakis J and Menendez J 2012 Appl. Phys. Lett. 101072105

[10] Bauer M, Taraci J, Tolle J, Chizmeshya A V G, Zollner S, Smith D J, Menendez J, Hu C and Kouvetakis J 2002 Appl. Phys. Lett. 812992

[11] Roucka R, Mathews J, Weng C, Beeler R, Tolle J, Menéndez J and Kouvetakis J 2011 IEEE J. Quantum Electron. 47213

[12] Roucka R, Beeler R, Mathews J, Ryu M Y, Kee Yeo Y, Menendez J and Kouvetakis J 2011 J. Appl. Phys. 109103115

[13] Werner J, Oehme M, Schirmer A, Kasper E and Schulze J 2012 Thin Solid Films 5203361

[14] Su S, Cheng B, Xue C, Wang W, Cao Q, Xue H, Hu W, Zhang G, Zuo Y and Wang Q 2011 Opt. Express 196400

[15] Zhang D, Xue C, Cheng B, Su S, Liu Z, Zhang X, Zhang G, Li C and Wang Q 2013 Appl. Phys. Lett. 102141111

[16] Su S, Wang W, Cheng B, Zhang G, Hu W, Xue C, Zuo Y and Wang Q 2011 J. Cryst. Growth 31743

[17] Sun G, Soref R A and Cheng H H 2010 J. Appl. Phys. 108 033107

[18] Yahyaoui N, Sfina N, Lazzari J-L, Bournel A and Said M 2013 Eur. Phys. J. B 86259

[19] Madelung O, Schultz M and Weiss H 1982 Physics of Group IV Elements and III-V Compounds 1st edn vol 17 (New York: Springer)

[20] Brudevoll T, Citrin D S, Cardona M and Christensen N E 1993 Phys. Rev. B 488629 
[21] Menéndez J and Kouvetakis J 2004 Appl. Phys. Lett. 851175

[22] Tonkikh A A, Eisenschmidt C, Talalaev V G, Zakharov N D, Schilling J, Schmidt G and Werner P 2013 Appl. Phys. Lett. 103032106

[23] Yahyaoui N, Sfina N, Lazzari J-L, Bournel A and Said M 2014 J. Appl. Phys. 115033109

[24] Millman J and Grabel A 1989 Dispositifs à Semiconducteurs (Paris: Ediscience International)

[25] Huang F Y, Zhu X, Tanner M O and Wang K L 1995 Appl. Phys. Lett. 67566

[26] Yamanishi M and Suemune I 1984 Jpn. J. Appl. Phys. 23 L35

[27] Sze S M 1981 Physics of Semiconductor Devices (New York: Academic)

[28] Sfina N, Lazzari J-L and Said M 2012 Superlattices Microstruct. 52901

[29] Ahn D and Chuang S L 1987 IEEE J. Quantum Electron. 232196

[30] Yahyaoui N, Sfina N, Abdi-Ben Nasrallah S, Lazzari J-L and Said M 2014 Comput. Phys. Commun. 1853119

[31] Ando Y and Itoh T 1987 J. Appl. Phys. 611497
[32] Berashevich J A, Danilyuk A L, Kholod A N and Borisenko V E 2003 Mater. Sci. Eng. B 101111

[33] Berashevich J A, Borisenko V E, Lazzari J-L and D'Avitaya F A 2007 Phys. Rev. B 75115336

[34] Sfina N, Yahyaoui N, Said M and Lazzari J-L 2007 Semicond. Sci. Technol. 22231

[35] Shim J, Liu B, Piprek J and Bowers J E 2004 IEEE Photon. Technol. Lett. 161474

[36] Masini G, Sahni S, Capellini G, Witzens J and Gunn C 2008 Advances in Optical Technologies 2008196572

[37] Gassenq A, Gencarelli F, Van Campenhout J, Shimura Y, Loo R, Narcy G and Roelkens G 2012 Opt. Express 20 27297

[38] Conley B R, Mosleh A, Ghetmiri S A, Soref R A, Sun G, Margetis J, Tolle J, Naseem H A and Yu S Q 2014 Opt. Express 2215639

[39] Ahn D, Hong C-Y, Liu J, Giziewicz W, Beals M, Kimerling L C, Michel J, Chen J and Kärtner F X 2007 Opt. Express 153916

[40] Kang Y et al 2009 Nat. Photonics 359

[41] Michel J, Liu J F and Kimerling L C 2010 Nat. Photonics 4527 